\title{
3D mapping of blood vessel networks and cells in COPD and non-COPD lung tissue
} samples using micro-computed tomography and immunofluorescence

M.J. Lawson ${ }^{1}$, O.L. Katsamenis ${ }^{2}$, M. Olding ${ }^{3}$, O. Larkin ${ }^{3}$, B. Smit ${ }^{3}$, I. Haig ${ }^{3}$, A. Alzetani ${ }^{1}$, P. Schneider ${ }^{4}$, P. Lackie ${ }^{1}$, J. Warner ${ }^{1}$

'Academic Unit of Clinical and Experimental Sciences, Faculty of Medicine, University of Southampton, Southampton, UK ${ }^{2} \mu$-VIS X-ray Imaging Centre, Faculty of Engineering and Physical Sciences, University of Southampton, Southampton, UK ${ }^{3}$ Nikon X-Tek Systems Ltt.

\section{Background}

Micro-Computed Tomography ( $\mu \mathrm{CT})$ is a non-destructive X-ray imaging technique used to visualise the 3D micro-structure of human lung tissue, this was combined with immunofluorescence to identify specific cells within a 3D volume

Microscopic changes in airways, vasculature networks and infiltrating cells are known to be features of lung diseases such as COPD but have not been quantified in 3D

\section{Aims}

1. Visualise the 3D networks and cells types in human lung tissue by registering and segmenting immunofluorescence (IF) to $\mu \mathrm{CT}$

2. Analyse the 3D networks of blood vessels in non-COPD and COPD lung tissue samples

3. Assess populations of specific cell types to quantify their relative location to blood vessels in 3D

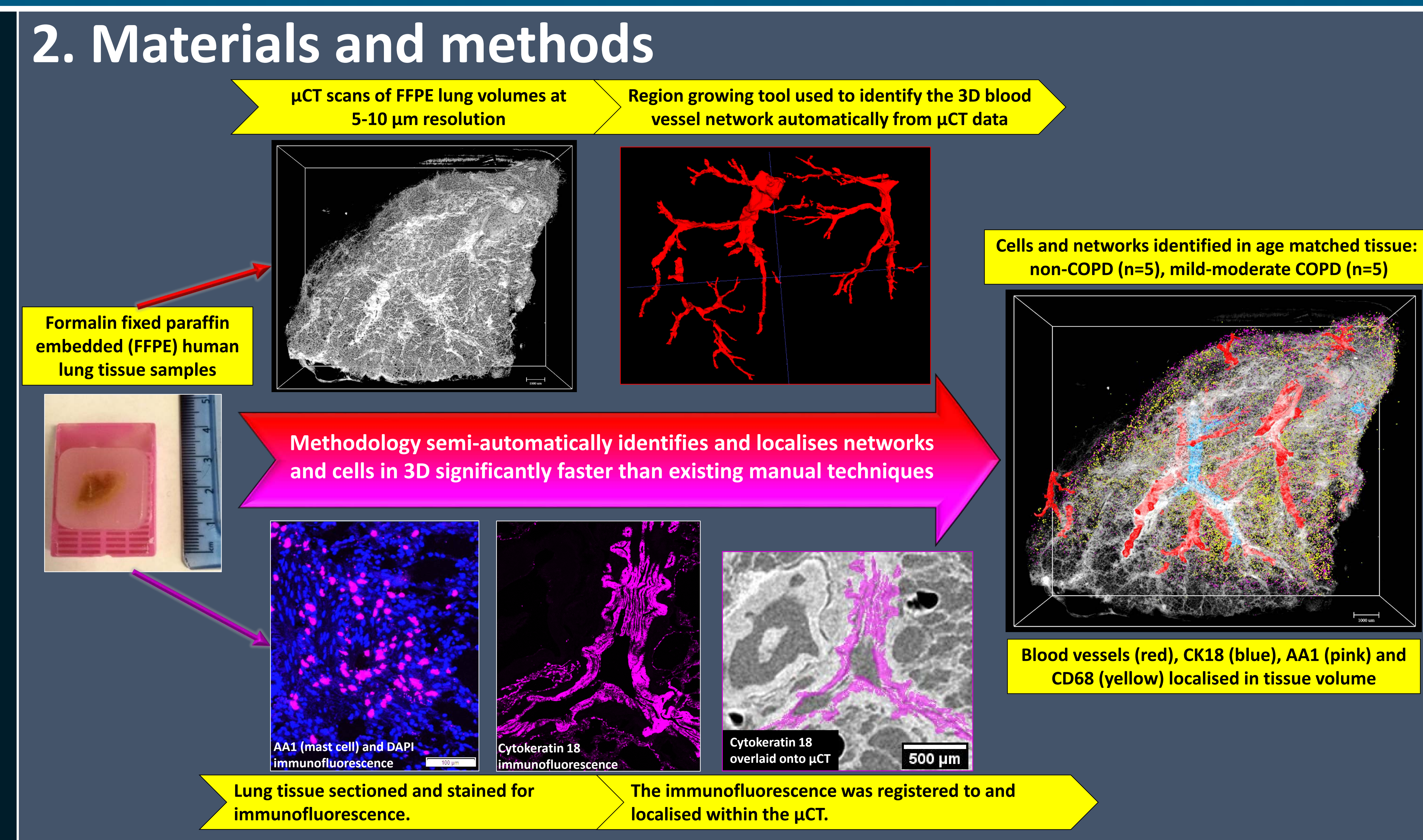

3. Identifying and analysing 3D blood networks

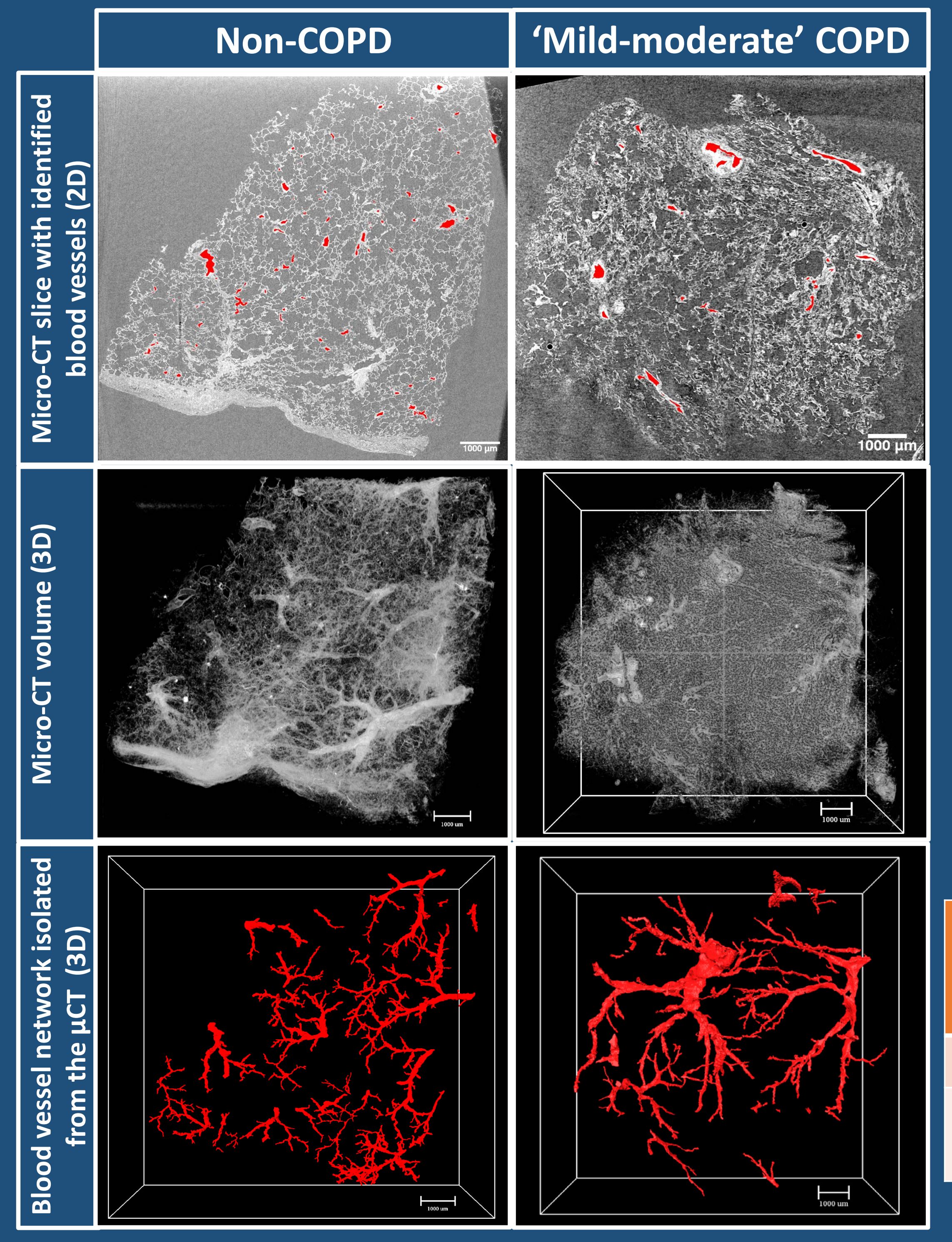

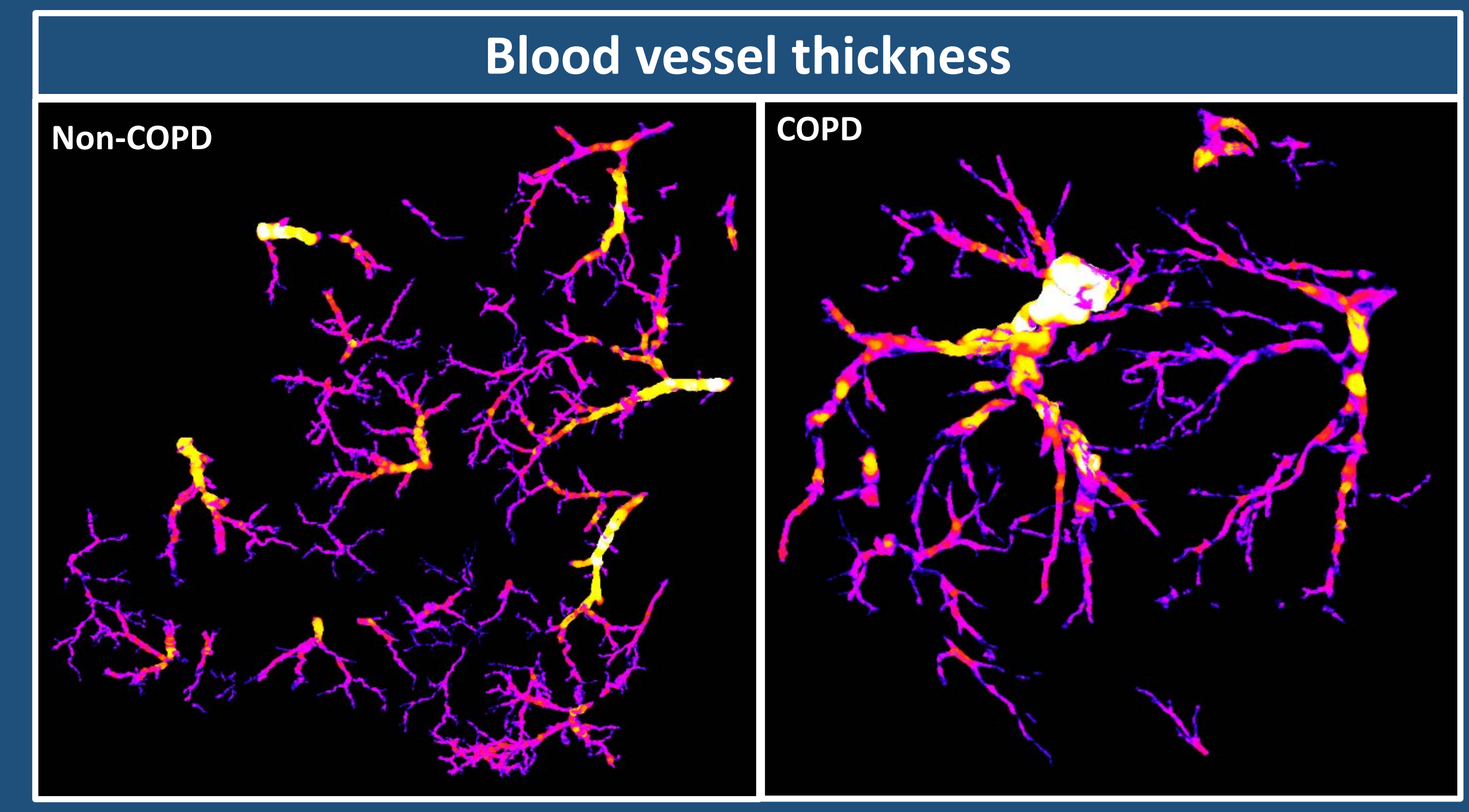

$>$ Thickness maps as a proxy for lumen diameter of blood vessel networks (brighter colour=thicker vessel)
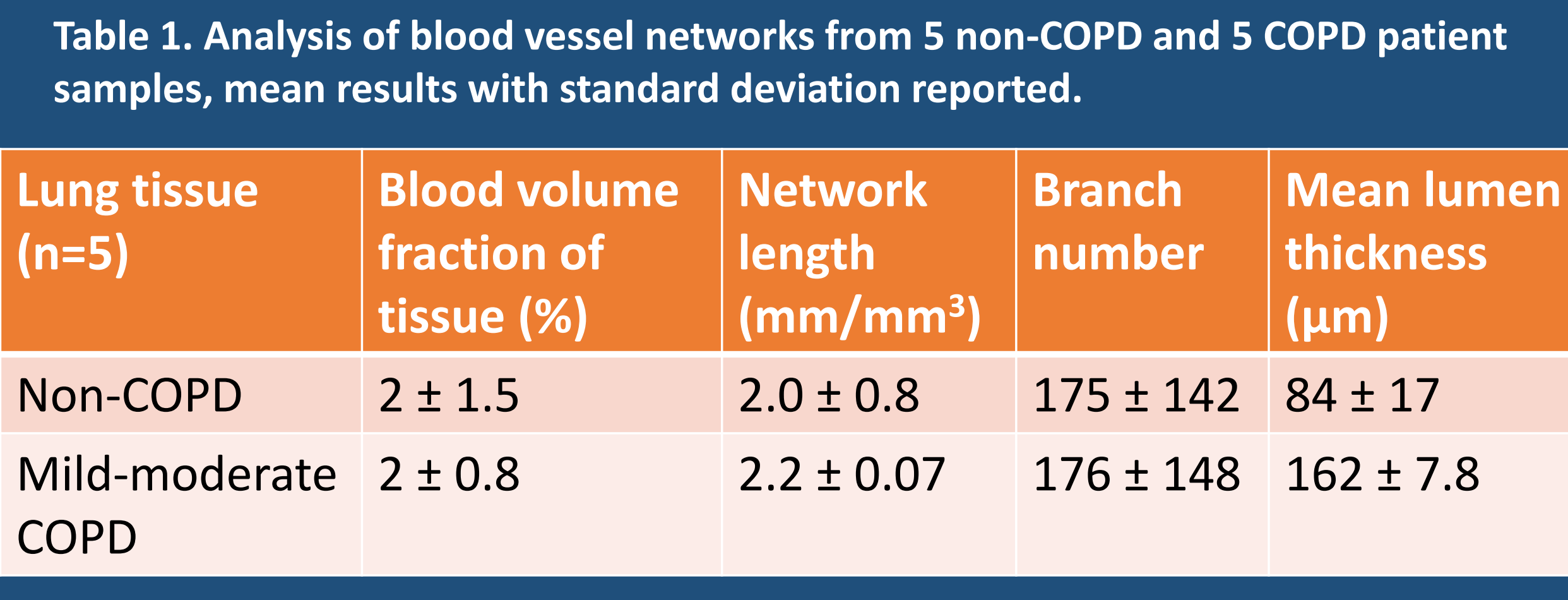

4. Correlative immunofluorescence and $\mu \mathrm{CT}$ imaging

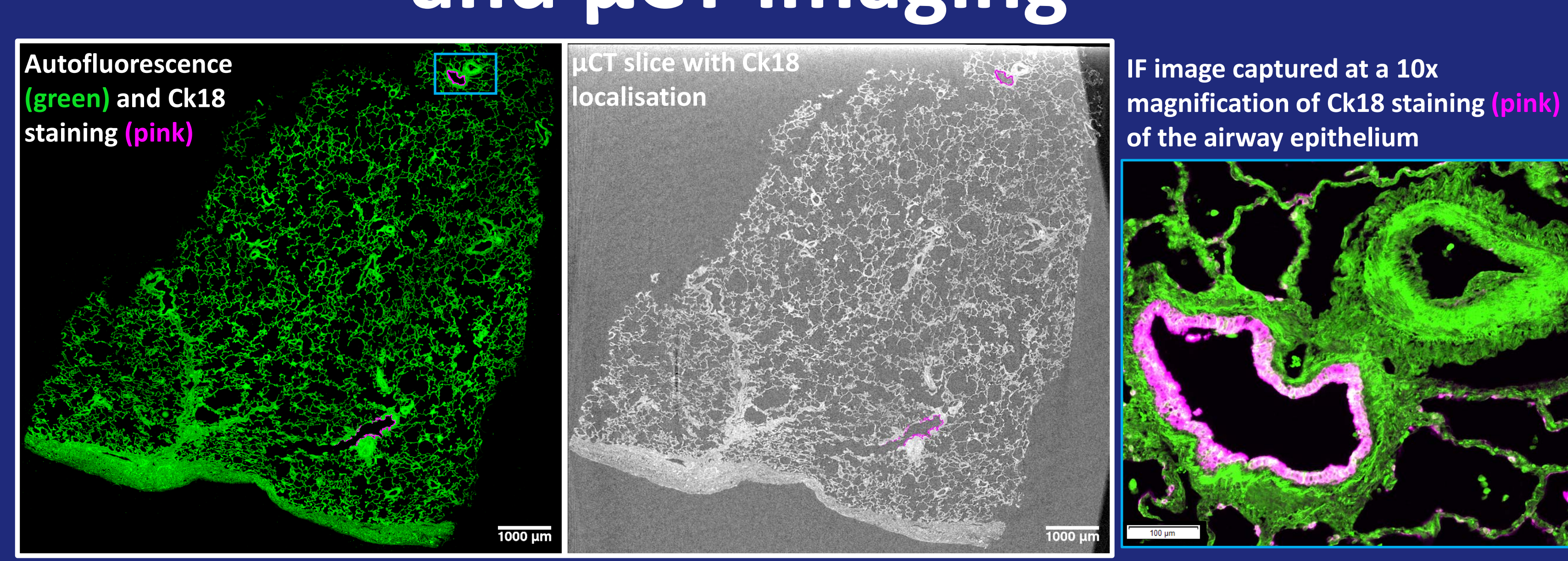

$>$ Registration of morphological distinct airway epithelium using Cytokeratin-18 validates the automated localisation of features identified by IF in the $\mu \mathrm{CT}$

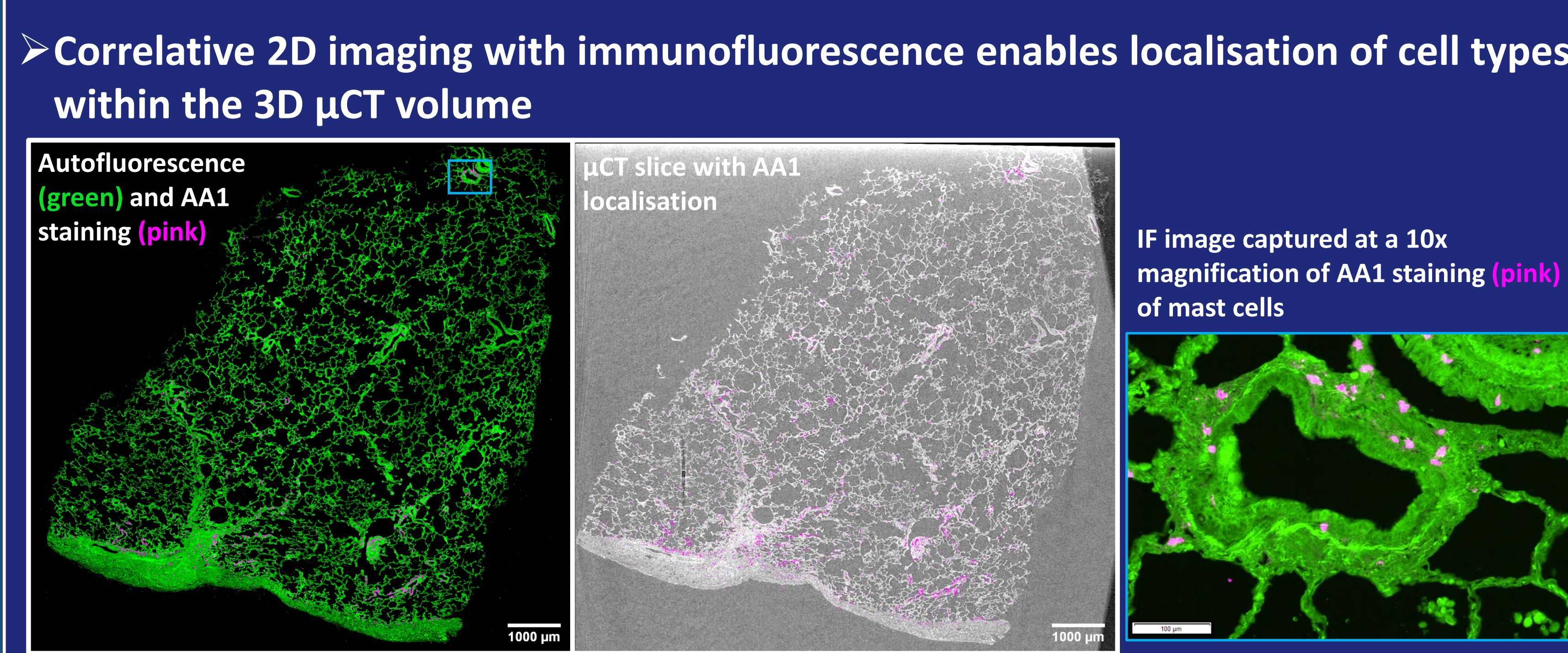

\section{Combining 3D network information with cellular staining} \begin{tabular}{|l}
\hline \\
\hline 2 \\
\hline
\end{tabular}
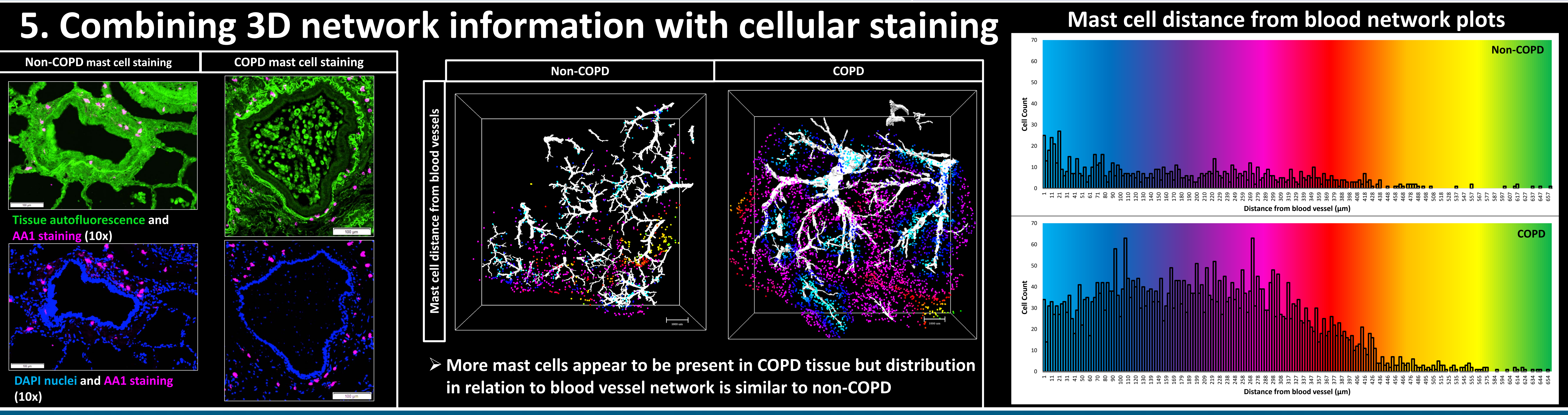

\section{Conclusions}

$>$ Micro-CT combined with immunofluorescence was successfully used to identify blood vessels, Ck18, macrophages and mast cells within the three-dimensional lung volume in both non-COPD and 'mild-moderate' COPD lung tissue samples

Analysis of the blood vessels suggests little difference between these networks in healthy and COPD lungs in 3D however there is great variability between samples in each group

\section{Acknowledgments}

$>$ Mast cells are widely distributed as individual cells throughout the tissue with a trend towards more mast cells being identified in COPD tissue

$>$ The distribution of mast cells in relation to blood vessels did not change between COPD and non-COPD with majority of mast cells located within $400 \mu \mathrm{m}$ of the nearest blood vessel in the 3D volume 